\title{
Etude théorique des performances de l'inverseur ECL à base de transistors bipolaires à hétérojonction GaAlAs/GaAs
}

\author{
J. Tasselli, A. Cazarre, J. P. Bailbe, A. Marty et G. Rey \\ Laboratoire d'Automatique et d'Analyse des Systèmes, 7 Avenue du Colonel Roche, 31077 Toulouse Cedex, \\ France
}

(Reçu le 20 octobre 1987, révisé le 11 janvier 1988, accepté le 22 février 1988)

\begin{abstract}
Résumé. - Dans cet article, nous rappelons les propriétés essentielles de la logique à émetteur couplé (E.C.L.) à base de transistors bipolaires à hétérojonction GaAlAs/GaAs. Nous présentons tout d'abord le modèle choisi pour simuler l'évolution du temps de propagation par porte en fonction de la puissance consommée. Une étude d'optimisation sur l'évolution du temps de propagation en fonction des valeurs typiques des principaux paramètres technologiques des transistors est ensuite effectuée. La simulation électrique d'un oscillateur en anneau a permis de calculer des temps de propagation par porte de 20 ps pour une puissance consommée de $4 \mathrm{~mW}$. Une comparaison avec l'état de l'art est enfin illustrée ; la comparaison avec les performances des circuits silicium montre l'intérêt de la filière bipolaire sur GaAs.
\end{abstract}

\begin{abstract}
In this paper, we first report the emitter coupled logic (E.C.L.) characteristics with GaAlAs/GaAs heterojunction bipolar transistors. Then, we present a theoretical study of the ECL potentialities, based on a ring oscillator simulation. The circuit behaviour sensitivity to the HBT's parameters variations is discussed. A propagation time of 20 ps for a $4 \mathrm{~mW}$ power dissipation have been obtained. At last, we confront our performances with the Si circuit's ones to show the interest of the GaAs bipolar branch.
\end{abstract}

\section{Introduction.}

Les transistors bipolaires à hétérojonction $\mathrm{Ga}$ $\mathrm{AlAs} / \mathrm{GaAs}$ ont fait l'objet de nombreuses études ces dernières années. Leurs potentialités ont été largement analysées par Kroemer [1].

Leurs performances fréquentielles $\left(F_{\max }\right.$ $=100 \mathrm{GHz}$ [2] ) et leur faible résistance de base en font des dispositifs très intéressants dans le domaine des circuits intégrés rapides. De plus, la structure bipolaire bénéficie d'un dimensionnement vertical qui lui confère une grande uniformité de tensions de seuil.

Ainsi, les transistors bipolaires à hétérojonction $\mathrm{GaAlAs} / \mathrm{GaAs}$ présentent un grand intérêt dans le domaine des circuits intégrés rapides. Ceci est un avantage considérable par rapport au dispositif à effet de champ pour lequel la réduction des dimensions horizontales est plus difficile.

L'étude de circuits ECL (logique à émetteur couplé) à partir de transistors bipolaires à hétérojonction a été menée [3,4]. A titre indicatif, des temps de propagation de l'ordre de 17 ps pour une puissance dissipée de $19 \mathrm{~mW}$ ont été obtenus sur un oscillateur en anneau en ECL [3].
Dans cet article, nous présentons les principaux critères d'optimisation d'une porte logique en ECL sur $\mathrm{AsGa}$, basée sur une étude de sensibilité aux paramètres technologiques du transistor bipolaire à hétérojonction.

\section{Propriétés de l'inverseur ECL sur GaAs.}

L'analyse et l'optimisation des circuits logiques ECL ont fait l'objet de nombreux travaux. Nous allons, ici, nous attacher à dégager les deux principaux critères de performance au niveau de la porte élémentaire que sont le temps de propagation $\tau_{\mathrm{pd}}$ et la puissance consommée $P d$ (le critère de qualité étant défini par le facteur de mérite $\left.P d \times \tau_{\mathrm{pd}}\right)$.

Le calcul rigoureux du temps de propagation d'une porte élémentaire ne peut être mené à bonne fin, compte tenu de la nature même du fonctionnement en régime de forts signaux d'un circuit logique, que par l'utilisation de programmes de simulation de circuits. Cependant, les analyses approchées, développées pour les études du transistor bipolaire, permettent de définir des caractéristiques semiquantitatives qui ont le mérite de mettre en évidence 
les principaux mécanismes électriques qui régissent le comportement de la structure.

Pour exemple, Tang et Solomon [5] ont proposé une relation semi-empirique, déduite d'une étude de sensibilité de $\tau_{\text {pd }}$ aux paramètres technologiques des transistors bipolaires, qui est donnée par :

$$
\begin{aligned}
\tau_{\mathrm{pd}}=k_{1} R_{1} C_{\mathrm{TC}}+ & k_{2} R_{1} C_{\mathrm{TE}}+k_{3} R_{1} C_{\mathrm{S}}+ \\
& +k_{4} R_{1} C_{\mathrm{L}}+k_{5} \tau_{\mathrm{B}}+k_{6} R_{\mathrm{bi}} C_{\mathrm{TC}}+ \\
& +k_{7} R_{\mathrm{bi}} C_{\mathrm{DE}_{\mathrm{m}}}+k_{8} R_{\mathrm{bx}} C_{\mathrm{DE}_{\mathrm{m}}}
\end{aligned}
$$

où la capacité de diffusion moyenne $C_{\mathrm{DE}_{\mathrm{m}}}$ est donnée, en utilisant le concept du contrôle de charge, par:

$$
C_{\mathrm{DE}_{\mathrm{m}}}=\frac{2 \tau_{\mathrm{B}} I_{\mathrm{C}}}{\Delta V_{\mathrm{L}}}
$$

$\Delta V_{\mathrm{L}}$ étant l'excursion logique en sortie de la porte, $I_{C}$ le courant collecteur et $\tau_{B}$ le temps de stockage des porteurs. $C_{\mathrm{TC}}$ et $C_{\mathrm{TE}}$ sont les capacités de transition base-collecteur et base-émetteur à polarisation nulle, $C_{\mathrm{L}}$ la capacité de charge de la porte, $C_{\mathrm{S}}$ la capacité de substrat, $R_{1}$ la résistance de collecteur telle que $R_{1_{\mathrm{C}}}=\frac{\Delta V_{\mathrm{L}}}{I}, R_{\mathrm{bx}}$ et $R_{\mathrm{bi}}$ désignent respectivement la résistance de base relative à la zone latérale et la résistance de base relative à la zone active (Fig. 1).

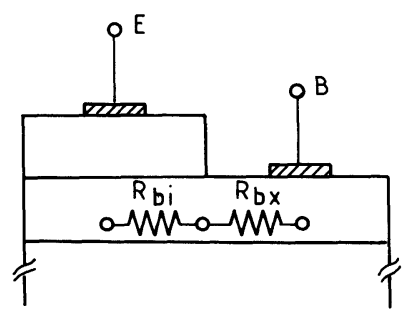

Fig. 1. - Représentation schématique de la résistance de base.

[Schematic picture of the base resistance.]

Cette formulation apparaît donc comme une somme de constantes de temps $\mathrm{RC}$, chacune pondérée par une constante $k_{\mathrm{i}}$ déterminée et propre à la topologie du circuit. Les effets Miller ainsi que la modulation des capacités de transition par les diverses tensions sont aussi pris en compte par les paramètres $k_{\mathrm{i}}$.

Dans le cas d'une excursion logique fixée, les asymptotes relatives à chacun des termes de propagation en fonction du courant collecteur $I_{C}$ (image de la puissance consommée par porte) qui sont reproduites sur la figure 2 appellent les commentaires suivants :

- les termes relatifs à $R_{1}$ décroissent quand le niveau de polarisation croît puisque $R_{1}$ décroît

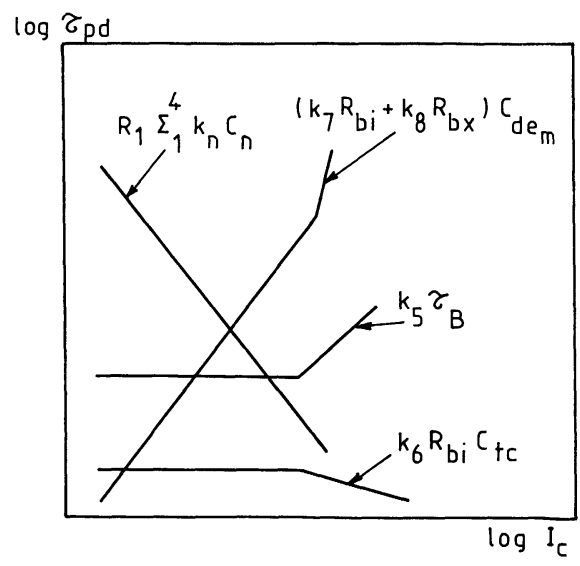

Fig. 2. - Asymptotes relatives aux différentes constantes de temps dans $\tau_{\mathrm{pd}}$.

[Asymptotic representation of the different delay times.]

lorsque $I_{C}$ augmente ; ces termes décroissent également quand les capacités de transition diminuent en même temps que les dimensions des transistors;

- les termes $R_{\mathrm{bi}} C_{\mathrm{DE}_{\mathrm{m}}}$ et $R_{\mathrm{bx}} C_{\mathrm{DE}_{\mathrm{m}}}$ augmentent avec le courant $\left(C_{\mathrm{DE}_{\mathrm{m}}} \alpha I_{\mathrm{C}}\right)$, et diminuent lorsque l'épaisseur de base $W_{\mathrm{B}}$ est réduite $\left(R_{\mathrm{b}} \alpha \frac{1}{W_{\mathrm{B}}}\right.$, $r_{\mathrm{B}} \alpha W_{\mathrm{B}}^{2}$ ) (Tab. I) ;

- aux faibles niveaux d'injection, les termes $R_{\mathrm{bi}} C_{\mathrm{TC}}$ et $\tau_{\mathrm{B}}$ varient faiblement avec le courant; $R_{\mathrm{bi}} C_{\mathrm{TC}}$ devient rapidement insignifiant quand la taille des transistors diminue.

Dans ces conditions, outre les avantages communs à toutes les structures bipolaires qui résident essentiellement dans le meilleur contrôle des dimensions verticales (pour la vitesse) et de la tension de seuil (pour l'intégration à large échelle), le transistor bipolaire à hétérojonction $\mathrm{GaAlAs} / \mathrm{GaAs}$ apporte des possibilités nouvelles.

Le temps de propagation $\tau_{\mathrm{pd}}$ sera plus petit pour une porte AsGa que pour une porte silicium. En effet, il est clair que les différentes constantes de temps qui interviennent dans la valeur de $\tau_{\mathrm{pd}}$ (relation (1)) sont inférieures dans le cas du GaAs : le temps de transit des électrons (inversement proportionnel à la mobilité) diminue ; la contribution liée au stockage des charges dans l'émetteur devient négligeable par suite de la très forte valeur de l'efficacité d'injection, qui limite sévèrement l'injection des trous dans cette zone; la capacité de transition émetteur-base décroît pour sa part en même temps que le dopage d'émetteur; enfin la résistance d'accès à la base est d'autant plus faible que le dopage de base est grand.

$\mathrm{Au}$ total, les très grandes possibilités des transistors bipolaires à hétérojonction doivent conférer à la logique ECL AsGa des potentialités que nous allons maintenant évaluer de façon précise. 
Tableau I.- Expressions des différents paramètres du modèle grands signaux du transistor.

[Typical parameters of the heterojunction transistor model.]

Efficacité d'injection : $\gamma=\frac{J_{1}}{J_{\mathrm{PBE}}}$

$$
\text { avec } J_{\mathrm{PBE}}=\frac{A_{\mathrm{E}} q \mu_{\mathrm{PE}} U_{\mathrm{T}}}{N_{\mathrm{DE}}\left(W_{\mathrm{E}}-W_{\mathrm{TE}}\right)} \exp \left(\frac{V_{\mathrm{BE}}}{U_{\mathrm{T}}}\right) \quad J_{1}=\frac{A_{\mathrm{E}} q n_{\mathrm{iB}}^{2} \mu_{\mathrm{nB}} U_{\mathrm{T}}}{N_{\mathrm{AB}} W_{\mathrm{B}}}\left(\exp \left(\frac{V_{\mathrm{BE}}}{U_{\mathrm{T}}}-1\right)\right)
$$

Capacités : $* C_{1}=C_{\mathrm{TE}}+C_{\mathrm{DE}}$

Résistances: $\quad R_{1}=R_{2}=1 \Omega$

$$
R_{3}=R_{\mathrm{BB}^{\prime}}=R_{\mathrm{bx}}+R_{\mathrm{bi}}
$$

avec

$$
C_{\mathrm{TE}}=\frac{A_{\mathrm{E}} \varepsilon_{\mathrm{E}}}{W_{\mathrm{TE}}}=A_{\mathrm{E}} \sqrt{\frac{q \varepsilon_{\mathrm{E}} N_{\mathrm{DE}}}{2\left(V_{\mathrm{DE}}-V_{\mathrm{BE}}\right)}}
$$$$
C_{\mathrm{DE}}=\left(\tau_{\mathrm{B}}+\frac{\tau_{\mathrm{SE}}}{\gamma}\right) \frac{J_{1}}{U_{\mathrm{T}}}+\tau_{\mathrm{C}} \frac{J_{2}}{U_{\mathrm{T}}} \quad \text { où } \quad \tau_{\mathrm{B}}=\frac{W_{\mathrm{B}}^{2}}{2 \mu_{\mathrm{nB}} U_{\mathrm{T}}}
$$$$
\tau_{\mathrm{SE}}=\frac{\left(W_{\mathrm{E}}-W_{\mathrm{TE}}\right)^{2}}{2 \mu_{\mathrm{pE}} U_{\mathrm{T}}}
$$

* $C_{2}=C_{\mathrm{TC}}=A_{\mathrm{E}} \sqrt{\frac{q N_{\mathrm{DC}} \varepsilon_{\mathrm{C}}}{2\left(V_{\mathrm{DC}}+V_{\mathrm{CB}}\right)}}$ $\tau_{\mathrm{C}}=\frac{W_{\mathrm{TC}}}{2 v_{1}}$

résistance de base $R_{\mathrm{BB}^{\prime}}=\frac{4}{3} \times \frac{1}{q \mu_{\mathrm{pB}} N_{\mathrm{AB}}} \times \frac{L}{W_{\mathrm{B}} L_{\mathrm{E}}}$ structure $S_{1 \mathrm{~B}}$ à simple contact de base

$$
R_{\mathrm{BB}^{\prime}}=\frac{7}{12} \times \frac{1}{q \mu_{\mathrm{pB}} N_{\mathrm{AB}}} \times \frac{L}{W_{\mathrm{B}} L_{\mathrm{E}}}
$$

structure $S_{2 B}$ à double contact de base

\section{Outil de simulation.}

La figure 3 présente le modèle grands signaux $\mathrm{du}$ transistor bipolaire à hétérojonction $\mathrm{GaAlAs}$ / GaAs qui constitue le fondement de nos simulations [6]. Utilisé ici pour simuler le comportement du transistor en régime normal, compte tenu de ce que l'ECL est une logique non saturée, il rend compte successivement :

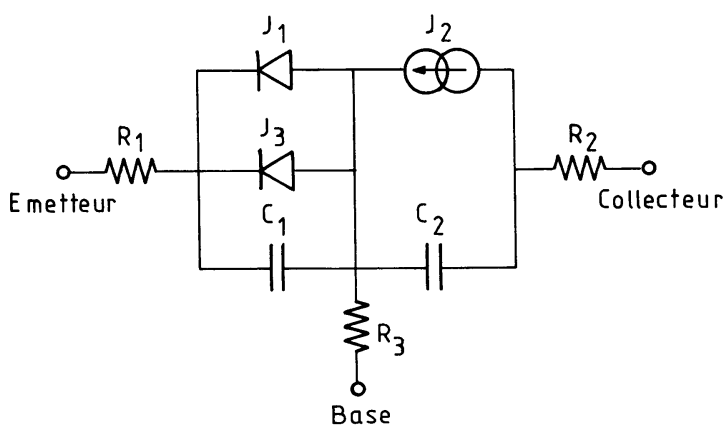

Fig. 3. - Schéma équivalent du transistor bipolaire à hétérojonction GaAlAs/GaAs.

[Equivalent circuit of the $\mathrm{GaAlAs} / \mathrm{GaAs}$ heterojunction bipolar transistor.]
- de la caractéristique de transfert $I_{\mathrm{C}}\left(V_{\mathrm{BE}}\right)$ calculée en prenant en compte les éventuels effets de la barrière de potentiel inhérente au spike de la bande de conduction dans la région d'émetteur,

- des mécanismes de recombinaison dans la région quasi neutre de base, par l'intermédiaire du facteur de transport dans la base $\delta$,

- de la composante du courant de recombinaisons $J_{3}$ dans la zone de transition émetteur-base,

- de l'influence des zones résistives de base $\left(R_{3}\right)$, de collecteur $\left(R_{2}\right)$ et d'émetteur $\left(R_{1}\right)$. La résistance de base rend compte à la fois de la zone latérale (extrinsèque) et de la zone active (intrinsèque),

- des capacités de transition des jonctions émetteur-base et base-collecteur, $C_{\mathrm{TE}}$ et $C_{\mathrm{TC}}$ respectivement, de la capacité de diffusion de la jonction baseémetteur, $C_{\mathrm{DE}}\left(C_{1}=C_{\mathrm{TE}}+C_{\mathrm{DE}} ; C_{2}=C_{\mathrm{TC}}\right)$,

- du temps de transit des porteurs dans la base $\left(\tau_{\mathrm{B}}\right)$, dans l'émetteur $\left(\tau_{\mathrm{E}}\right)$ et dans la zone de transition base-collecteur $\left(\tau_{C}\right)$.

Pour évaluer les potentialités de la filière ECL AsGa à base de TBH AsGa, nous avons opté pour une caractérisation qui s'appuie sur les résultats de la 


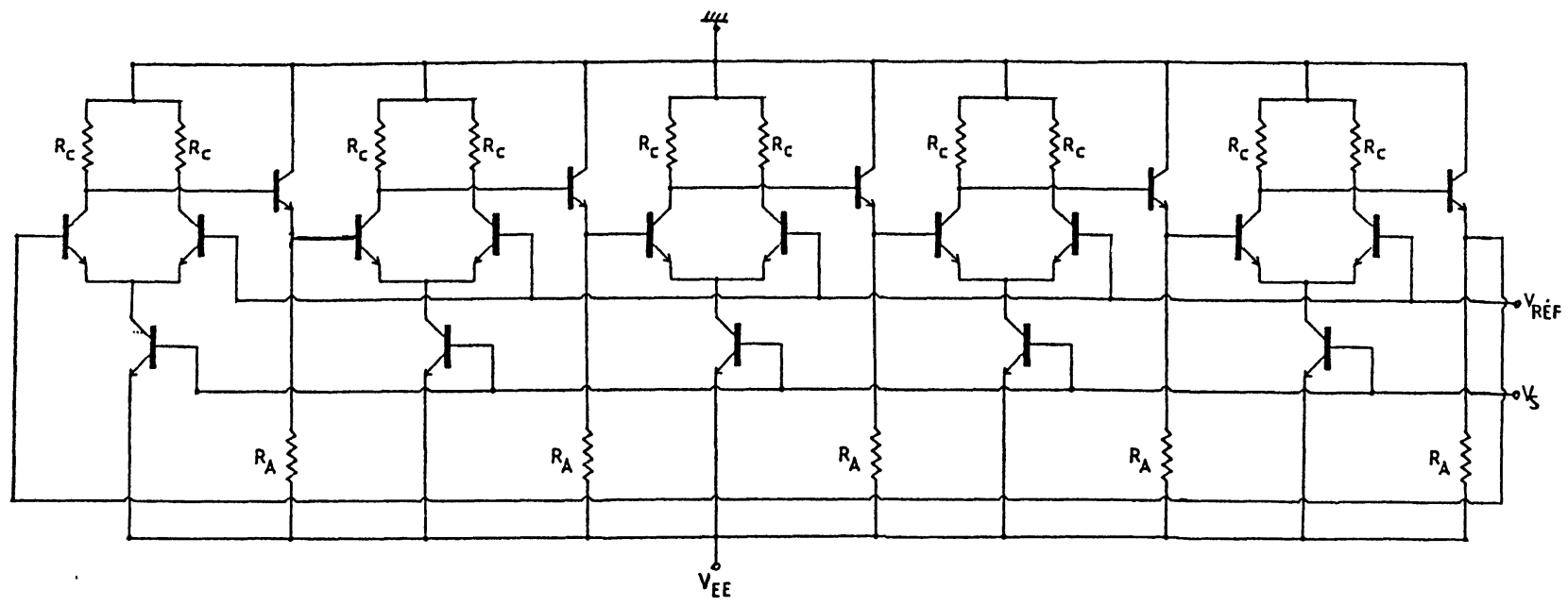

Fig. 4. - Schéma électrique de l'oscillateur en anneau à 5 portes ECL.

[Circuit schematic diagramm of the ring oscillator using five ECL inverters.]

simulation d'un oscillateur en anneau (Fig. 4), l'inverseur simulé est de type ECL avec émetteur suiveur.

En effet, ce circuit procure un moyen convenable et largement utilisé pour mesurer le temps de propagation en fonction de la puissance consommée [7]. Une chaîne constituée par un nombre impair $N$ d'inverseurs ou de portes logiques, est connectée en anneau ; la fréquence d'oscillation $f$ du circuit est reliée au temps de propagation $\tau_{\mathrm{pd}}$ de la porte élémentaire par la relation

$$
f=\frac{1}{2 N \tau_{\mathrm{pd}}} .
$$

La simulation du fonctionnement de l'oscillateur en anneau, à partir du simulateur de circuits ASTEC III, consiste en un examen du régime transitoire sur un temps $T_{\max }$ compatible avec l'obtention du régime oscillant stabilisé. La figure 5 illustre la réponse transitoire de l'oscillateur ainsi que les paramètres électriques typiques utilisés dans la simulation.

Nous nous proposons d'évaluer ici les critères de qualité de la porte ECL élémentaire à base de transistors à hétérojonction $\mathrm{GaAlAs} / \mathrm{GaAs}$, en analysant successivement l'influence des paramètres technologiques. Pour ce faire, nous adopterons un dimensionnement horizontal et vertical des structures raisonnable, compatible avec la maîtrise des techni-

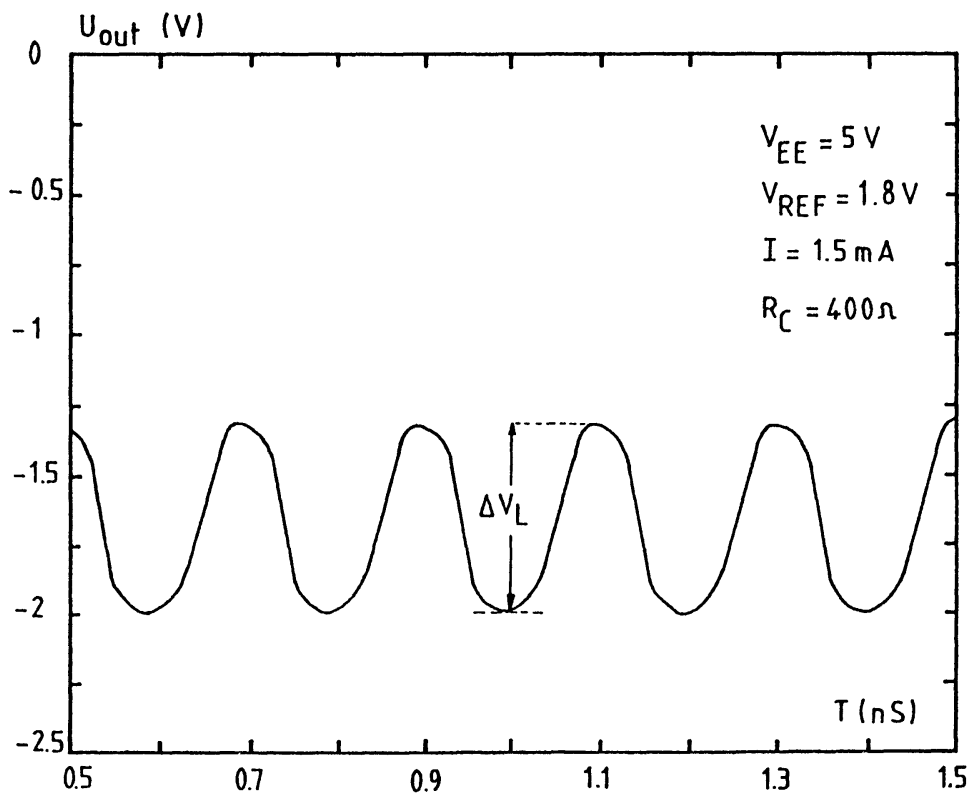

Fig. 5. - Réponse transitoire de l'oscillateur en anneau obtenue par simulation.

[Simulated transient output signal of the ring oscillator.] 
ques de lithographie et de croissance de couches actuelles.

L'ensemble des relations entre les paramètres physiques (de type dopage et largeurs) et les paramètres électriques correspondants est rassemblé dans le tableau I.

En ce qui concerne les dimensions superficielles, nous avons opté pour une règle de dessin $1 \mu \mathrm{m}$, ce qui signifie que la largeur des doigts de prise de contact d'émetteur et de base ainsi que l'espacement entre ces doigts est fixée à $1 \mu \mathrm{m}$. Il est clair, ici, que l'évaluation que nous allons proposer ne correspond pas à la limite théorique des performances que l'on pourrait calculer; dès lors, les progrès technologiques qu'il est permis d'espérer devraient permettre la réalisation de structures encore plus fines et donc plus performantes.

Tableau II. - Paramètres types utilisés dans le modèle du transistor à hétérojonction.

[Typical parameters of the heterojunction transistor model.]

\begin{tabular}{|c|c|c|}
\hline Emetteur & Base & Collecteur \\
\hline$N_{\mathrm{DE}}=10^{17} \mathrm{~cm}^{-3}$ & $N_{\mathrm{AB}}=10^{19} \mathrm{~cm}^{-3}$ & $N_{\mathrm{DC}}=10^{16} \mathrm{~cm}^{-3}$ \\
$W_{\mathrm{E}}=1 \mu \mathrm{m}$ & $W_{\mathrm{B}}=0,2 \mu \mathrm{m}$ & $W_{\mathrm{C}}=4 \mu \mathrm{m}$ \\
$X_{\mathrm{AIE}}=0,4$ & - & \\
$L_{\mathrm{GE}}=500 \AA$ & & \\
\hline
\end{tabular}

L'ensemble des valeurs initiales et typiques des paramètres du transistor, autour duquel va s'articuler notre étude de sensibilité est reporté dans le tableau II. Ces données appellent les commentaires suivants :

- le taux d'aluminium $X_{\mathrm{AlE}}=0,4$ et le dopage d'émetteur $N_{\mathrm{DE}} \approx 10^{17} \mathrm{~cm}^{-3}$ garantissent une forte efficacité d'injection qui permet de minimiser le stockage de charges dans cette région, et assurent un bon compromis pour la valeur de la capacité de transition de la jonction émetteur-base ;

- l'épaisseur de base $W_{\mathrm{B}}=0,2 \mu \mathrm{m}$ conduit à une faible résistance de base si l'on impose le dopage dans cette région prédéterminée à son niveau maximum acceptable : $N_{\mathrm{AB}}=10^{19} \mathrm{~cm}^{-3}$;

- le dopage $N_{\mathrm{DC}}$ et l'épaisseur $W_{\mathrm{C}}$ de la région de collecteur sont fixés selon les critères de tenue en tension inverse et de minimisation de la capacité de transition base-collecteur et de la résistance de la zone active de collecteur.

Sur la base des considérations qui viennent d'être discutées, nous allons successivement examiner l'influence de la résistance de base, à la fois rendue par une variation de la topologie de la prise de contact (structure à simple ou à double contact de base), ou bien par une modification de l'épaisseur de base, et l'influence des dopages des régions d'émetteur et de collecteur.

\section{Optimisation.}

4.1 INFLUENCE DE LA STRUCTURE DE PRISE DE CONTACT DE BASE. - La quasi-totalité des réalisations ECL Silicium ainsi que les premières études relatives à la filière AsGa mettent en œuvre une structure à double contact de base, ceci pour minimiser l'impact de la résistance de base qui peut être une des principales causes de la limitation des performances.

Il nous paraît intéressant d'examiner, ici, si cette contrainte ne peut être levée compte tenu du net avantage apporté par la possibilité de surdoper la région de base dans le transistor à hétérojonction. Pour ce faire, nous allons comparer les performances calculées pour les structures à un ou deux doigts de base.

Les géométries ainsi que les paramètres retenus pour cette confrontation sont rapportés sur la figure 6 et le tableau III.
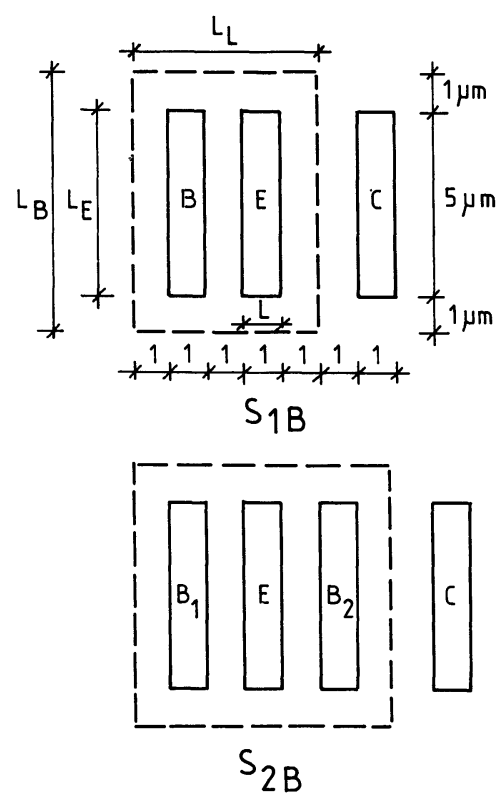

Fig. 6. - Deux types de géométrie utilisés: simple contact base $\left(S_{1 B}\right)$ et double contact base $\left(S_{2 B}\right)$.

[Two configurations : one base contact $\left(\mathrm{S}_{1 \mathrm{~B}}\right)$ and double base contact $\left(S_{2 B}\right)$.]

Les résultats de la simulation numérique qui permettent de calculer les variations du temps de propagation $\tau_{\mathrm{pd}}$ en fonction de la puissance consommée par porte, $P d$, sont reportés sur la figure 7 . Pour chacun des points de calcul, l'excursion logique $\Delta V_{\mathrm{L}}$ est fixée à $600 \mathrm{mV}$ et la tension d'alimentation à $5 \mathrm{~V}$. On relève une amélioration de $\tau_{\mathrm{pd}}$ dans pratiquement toute la gamme des puissances consommées 
Tableau III. - Paramètres relatifs aux deux géométries étudiées.

[Design of the two samples studied.]

\begin{tabular}{|l|c|c|}
\hline & $\mathrm{S}_{1 \mathrm{~B}}$ & $\mathrm{~S}_{2 \mathrm{~B}}$ \\
\hline$L$ & $1 \mu \mathrm{m}$ & $1 \mu \mathrm{m}$ \\
$L_{\mathrm{E}}$ & $5 \mu \mathrm{m}$ & $5 \mu \mathrm{m}$ \\
$L_{\mathrm{B}}$ & $7 \mu \mathrm{m}$ & $7 \mu \mathrm{m}$ \\
$L_{\mathrm{L}}$ & $5 \mu \mathrm{m}$ & $7 \mu \mathrm{m}$ \\
\hline$A_{\mathrm{E}}$ & $5 \mu \mathrm{m}_{2}$ & $5 \mu \mathrm{m}^{2}$ \\
$A_{\mathrm{C}}$ & $35 \mu \mathrm{m}^{2}$ & $49 \mu \mathrm{m}^{2}$ \\
$R_{\mathrm{BB}^{\prime}}$ & $102 \Omega$ & $45 \Omega$ \\
\hline
\end{tabular}

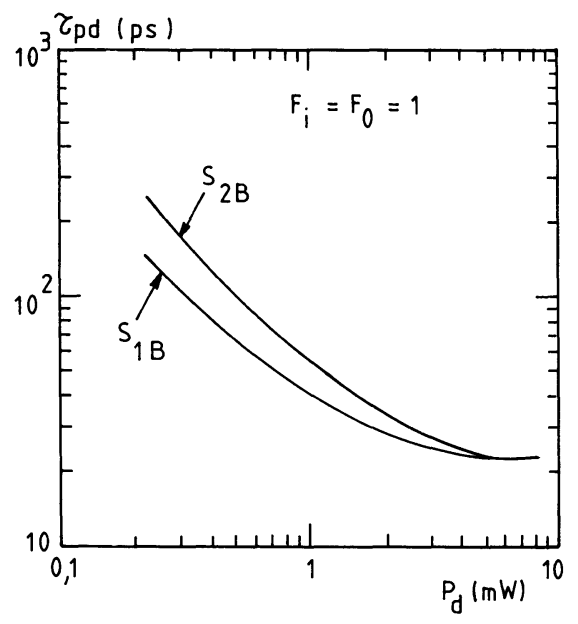

Fig. 7. - Variation du temps de propagation en fonction de la géométrie des transistors.

[Propagation time versus transistors topologie.]

explorée pour la structure à un doigt de base de plus petites dimensions.

Ce résultat met bien en évidence l'intérêt qu'il y a à réduire l'influence de la capacité de transition base-collecteur au détriment de l'augmentation de la résistance de base, et il semble donc que, pour une porte ECL sur GaAs, on doit favoriser le choix d'une structure à simple contact de base.

Il faut cependant noter qu'une neutralisation de la zone passive de collecteur située sous le ou les contacts de base, tels que l'ont proposé ASBECK et al ; en utilisant soit une implantation profonde d'oxygène [8], soit une implantation de protons [3], doit permettre de bénéficier de l'avantage apporté par la structure à deux doigts de base.

Il s'agit là d'un raffinement technologique qui, s'il s'avère être facilement maîtrisable dans l'élaboration de circuits intégrés, devra évidemment être pris en considération pour l'optimisation de la filière ECL sur GaAs.
4.2 INFLUENCE DE L'ÉPAISSEUR DE BASE. - La diminution de l'épaisseur de base entraîne une augmentation de la résistance de base $R_{\mathrm{BB}}$, et une diminution du temps de transit $\tau_{\mathrm{B}}$ des porteurs dans la base. Les résultats reportés sur la figure $6 \cdot \mathrm{mon}$ trent, qu'au bout du compte, on aboutit à une amélioration du temps de propagation dans toute la gamme de puissance consommée explorée, lorsque l'épaisseur de base diminue jusqu'à une limite proche de $0,1 \mu \mathrm{m}$. Il convient donc de choisir une base relativement mince.

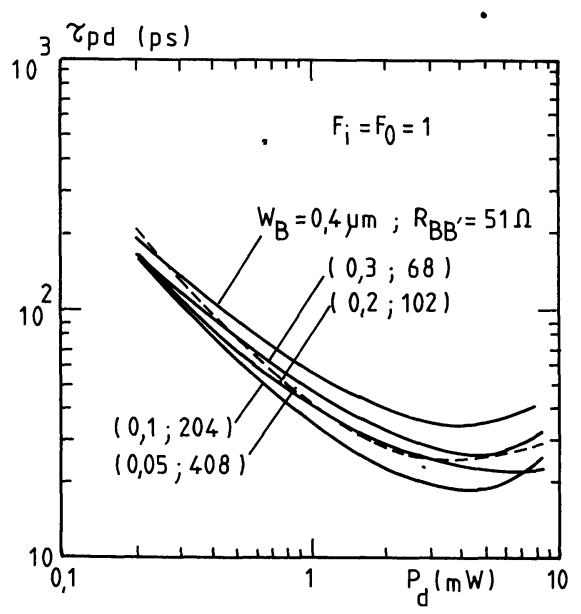

Fig. 8. - Variation du temps de propagation $\tau_{\mathrm{pd}}$ avec l'épaisseur de base $W_{\mathrm{B}}$.

[Propagation time $\tau_{\mathrm{pd}}$ versus base layer thickness.]

4.3 INFLUENCE DU DOPAGE D'ÉMETTEUR. - La figure 9 rend compte de la sensibilité du temps de propagation à la variation du dopage et de l'extension de la gradualité de la zone d'émetteur.

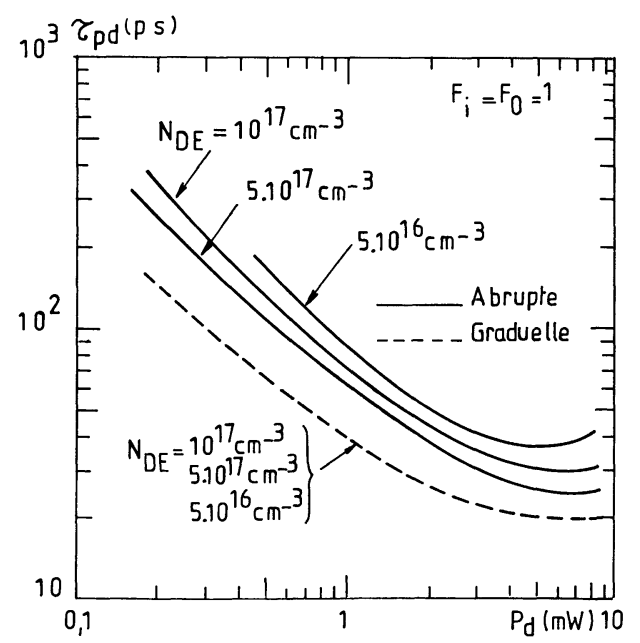

Fig. 9. - Variation du temps de propagation $\tau_{\mathrm{pd}}$ avec le dopage d'émetteur $N_{\mathrm{DE}}$.

[Propagation time $\tau_{\mathrm{pd}}$ versus emitter concentration $N_{\mathrm{DE}}$. 
Les paramètres liés aux caractéristiques de l'émetteur et influant sur le temps de propagation sont les capacités de transition $C_{\mathrm{TE}}$ et de diffusion $C_{\mathrm{DE}}$.

Que la jonction émetteur-base soit abrupte ou graduelle, l'influence de la capacité de transition $C_{\mathrm{TE}}$ est négligeable comparée à celle de la capacité de diffusion $C_{\mathrm{DE}}$ (voir Fig. 10).

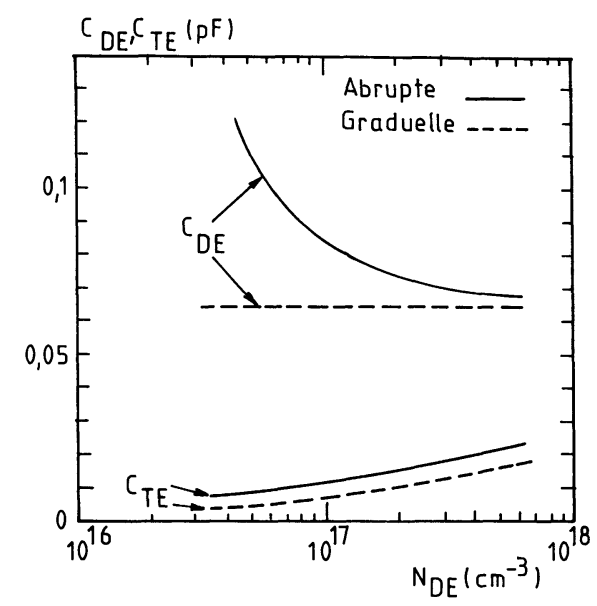

Fig. 10. - Variation des capacités de diffusion $C_{\mathrm{DE}}$ et de transition $C_{\mathrm{TE}}$ en fonction du dopage d'émetteur $N_{\mathrm{DE}}$.

[Variation of diffusion and transient capacitance $C_{\mathrm{DE}}$ and $C_{\mathrm{TE}}$ versus emitter concentration.]

Dans le cas d'une hétérojonction abrupte, l'efficacité d'injection prend des valeurs beaucoup plus faibles que dans le cas graduel ; le terme lié au temps de stockage des porteurs dans l'émetteur n'est donc plus négligeable devant le temps de transit dans la base $\tau_{\mathrm{B}}$ et induit une importante augmentation de $C_{\mathrm{DE}}$.

Ainsi, afin de diminuer le temps de propagation $\tau_{\mathrm{pd}}$, il faut réaliser des hétérojonctions émetteurbase graduelles ; un degré de liberté supplémentaire est alors laissé pour le choix du dopage d'émetteur.

4.4 INFLUENCE DU DOPAGE DE COLLECTEUR. - La capacité de transition $C_{\mathrm{TC}}$, le temps de transit des porteurs dans la zone de transition et la valeur limite de la densité de courant $J_{\mathrm{BWC}}$ au-delà de laquelle se manifeste l'élargissement de base dans le collecteur (Effet Kirk) sont les trois principaux paramètres électriques liés aux caractéristiques de la région de collecteur, qui contribuent à la limitation du comportement dynamique des transistors dans la porte ECL (ces circuits logiques fonctionnant à très basse tension, le problème de la tenue en tension ne se pose pas). Afin de supprimer l'effet Kirk, on doit augmenter la densité de courant collecteur selon l'expression $J_{\mathrm{BWC}}=q N_{\mathrm{DC}} V_{1}$ où $V_{1}$ désigne la vitesse limite.

Les résultats de l'étude de sensibilité au dopage de la région de collecteur sont reportés sur la figure 11. L'augmentation du temps de propagation avec le

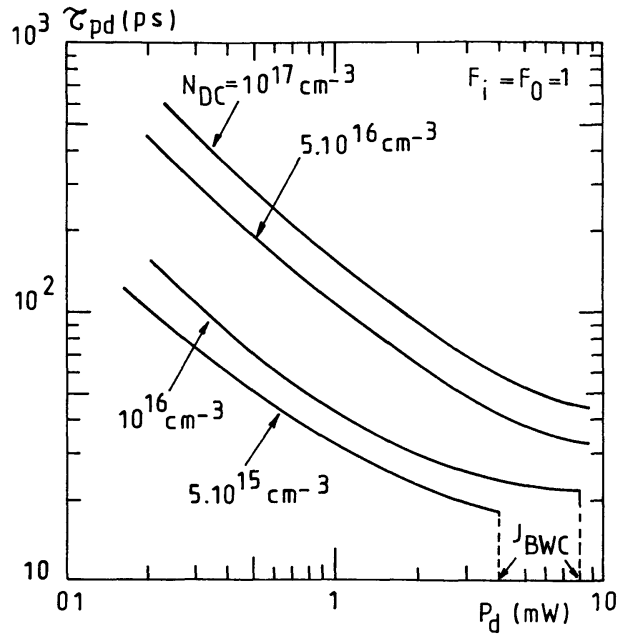

Fig. 11. - Variation du temps de propagation $\tau_{\mathrm{pd}}$ avec le dopage de collecteur $N_{\mathrm{DC}}$.

[Propagation time evolution versus concentration.]

dopage de collecteur est liée à l'augmentation de la capacité de transition $C_{\mathrm{TC}}$.

Bien que dans le cas de dopages faibles, le courant limite lié aux phénomènes d'élargissement de base apparaisse pour des puissances maximales consommées moindres que dans le cas des forts dopages, les meilleures performances sont tout de même obtenues pour des faibles valeurs de concentration.

\subsection{COMPARAISON DES PERFORMANCES OBTENUES} EN LOGIQUe ECL GaAs ET Si. - La figure 12 représente l'évolution du temps de propagation en fonction de la puissance dissipée pour les logiques ECL GaAs et silicium. Elle montre les potentialités de la logique ECL GaAs : en effet, sont comparés sur cette figure des résultats de simulation (courbes 12a et b) mais aussi des résultats expérimentaux.

En ce qui concerne les courbes théoriques, la comparaison a été faite entre les résultats de notre simulation (12b) et les performances prévues par ASBECK (12a). Celles-ci ont été obtenues à partir de transistors optimisés possédant des doigts d'émetteur de $1 \mu \mathrm{m}$ de large. L'amélioration obtenue $\left(\tau_{\mathrm{pd}}=20 \mathrm{ps}\right.$ pour une puissance consommée de $1 \mathrm{~mW}$ au lieu de 35 ps dans notre cas) confirme que pour augmenter les performances du circuit, il. convient de réduire les dimensions latérales des transistors par une amélioration des techniques de lithographie, l'utilisation du processus d'auto-alignement et l'emploi de l'implantation ionique pour éliminer les zones parasites : implantation profonde d'oxygène ou de proton effectuée sous les contacts de base, afin de réduire la capacité extrinsèque basecollecteur [3].

En ce qui concerne les circuits ECL réalisés à ce jour, les meilleurs temps de propagation ont été obtenus pour le GaAs par le Laboratoire NTT [9], à savoir 17,2 ps avec des transistors d'aire d'émetteur 


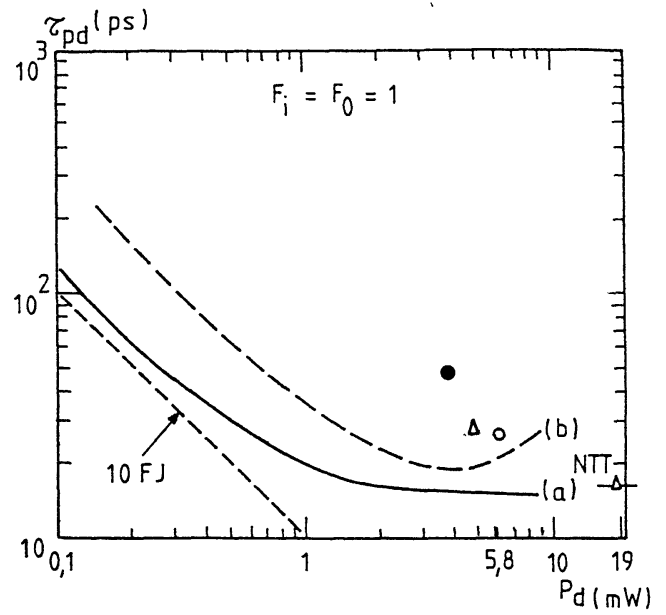

$\operatorname{GaAs}\left[\begin{array}{c}\text { - Simulation ASBECK : } \\ L=1 \mu \mathrm{m}\left\{\begin{array}{l}A_{\mathrm{E}}=(1 \times 5) \mu \mathrm{m}^{2} \\ \text { Réduction des zones } \\ \text { latérales (a) }\end{array}\right. \\ - \text { - Notre simulation (b) } ; A_{\mathrm{E}}=(1 \times 5) \mu \mathrm{m}^{2} \\ \Delta \quad \text { Réalisation NTT : } A_{\mathrm{E}}=(2 \times 5) \mu \mathrm{m}^{2} \\ \tau p d=17,2 \mathrm{ps} P d=19 \mathrm{~mW} \\ 0 \text { Asbeck : } \begin{array}{c}(2,5 \times 4,5) \mu \mathrm{m}^{2} d=27,6 \mathrm{ps} \\ P d=5,8 \mathrm{~mW}\end{array}\end{array}\right.$

$$
\mathrm{Si}\left[\begin{array}{cc}
\triangle & \begin{array}{c}
\text { Simulation Fairchild SAPT } A_{\mathrm{E}}=1 \mu \mathrm{m}^{2} \\
\tau_{\mathrm{pd}}=30 \mathrm{ps}
\end{array} \\
\bullet & \text { Simulation Hitachi } A_{\mathrm{E}}=(2 \times 3) \mu \mathrm{m}^{2} \\
\tau_{\mathrm{pd}}=48 \mathrm{ps} I=1 \mathrm{~mA}
\end{array}\right.
$$

Fig. 12. - Comparaison des logiques ECL GaAs et $\mathrm{Si}$ (simulation et expérience).

[Comparative GaAs and Si ECL logics (simulation and experiment results).]
$(2 \times 5) \mu \mathrm{m}^{2}$ conçus à partir d'une technologie autoalignée. Il faut aussi noter la réalisation d'un diviseur de fréquence fonctionnant à $22 \mathrm{GHz}$ publiée récemment [9]. Pour les circuits ECL sur silicium les meilleures performances à ce jour sont celles de Fairchild sur une technologie SAPT (30 ps/porte sur un oscillateur en anneau avec une technologie de $1 \mu \mathrm{m})$ [10] et de Hitachi avec $48 \mathrm{ps} /$ porte pour des surfaces d'émetteur de $(2 \times 3) \mu^{2}$ [11].

Malgré une maîtrise technologique moins au point, les circuits intégrés ECL sur GaAs s'avèrent être plus rapides que sur $\mathrm{Si}$. Ceci confirme nos propos sur les potentialités et avantages de cette filière logique, et laisse présager un développement futur de ce type de circuit.

\section{Conclusion.}

Cet article a été consacré à l'étude des potentialités de l'inverseur ECL à base de transistors bipolaires à hétérojonction GaAlAs/GaAs (HBT), à partir de la simulation d'un oscillateur en anneau à 5 portes. Les diverses propriétés de ce circuit ont été analysées.

Une étude de sensibilité du temps de propagation par porte $\tau_{\mathrm{pd}}$ aux principaux paramètres technologiques (dopage d'émetteur et de collecteur, épaisseur de base, géométrie des contacts) a permis de définir des critères d'optimisation. Un temps de propagation $\tau_{\text {pd }}$ de 20 ps pour une puissance consommée de $4 \mathrm{~mW}$ a été obtenue. Rappelons que ces circuits profitent à la fois de caractéristiques propres aux structures bipolaires (dimensionnement vertical et uniformité de la tension de seuil) et des caractéristiques des hétérojonctions GaAlAs/GaAs.

Ces résultats laissent présager un avenir prospère aux circuits intégrés bipolaires sur GaAs.

\section{Bibliographie}

[1] Kroemer, H., Heterostructure bipolar transistors and integrated circuits, Proc. IEEE 70 (1982) 1325.

[2] AsBeCK, P. M., ChANG, M. F. et al., Heterojunction Bipolar Transistor for Microwave and Millimeter-wave integrated circuits, IEEE 1987 Microwave and Millimeter-wave Monolithic Circuits Symposium, pp. 1-5.

[3] Ishibashi, T., Yamauchi, Y. et Nakajima, O., High Speed Frequency Dividers Using Self-aligned AlGaAs/GaAs Heterojunction Bipolar Transistors, IEEE, El. Device Lett. 8 (1987) 194196.

[4] Asbeck, P. M., Miller, D. L. et al., Emitter Coupled logic circuits implemented with heterojunction bipolar transistors, CaAs IC Symposium Technical Syst. (1983) pp. 170-173.
[5] TANG, D. D. et Solomon, P. M., Bipolar transistor design for optimized power delay logic circuits, IEEE J. Solid State Electron. SC 14 (1979) 679684.

[6] Marty, A., Rey, G. et Bailbe, J. P., Electrical Behavior of an NpN GaAlAs/GaAs heterojunction transistor Solid State Electron. 22 (1979) 549-557.

[7] Kurata, M. et KuTOH, R., Ring-oscillator-circuit simulation with physical model for GaAs/.GaAlAs H.B.T. IEEE Trans. Electron. Devices 32 (1985) 1086-1091.

[8] Asbeck, P. M., Miller, D. L. et Anderson, R. J., GaAs/GaAlAs HBT with buried oxygen implanted isolation layers IEEE Electron. Device Lett. 5 (1984) 310-312. 
[9] Chang, M. F., Asbeck, P. M., WANG, K. C. et al., AlGaAs/GaAs Heterojunction Bipolar transistor circuits with improved high-speed performance Electron. Lett. 22 (1986) 1173-1174.

[10] Yamauch, Y., Nagata, K. et al., $22 \mathrm{GHz} 1 / 4$ Frequency Divider using AlGaAs/GaAs HBTs, Electron. Lett. 23 (1987) 881-882.
[11] Vora, M., Ho, Y. L., Bhamre, S., Chien, F., BAKKer, G., HiNGARH, H. et SCHMITZ, C., Fairchild Research Center, A SUB 100 Picosecond Bipolar ECL Technology, IEDM (1985) pp. 34-37.

[12] HITACHI, ISSCC (1987) pp. 58-59. 